\title{
3 Research Soure \\ Tuning Iron Spin State in Single-Atom Nanozymes Enables Efficient Peroxidase Mimicking
}

\section{Xiaoqian Wei}

Central China Normal University

\section{Shaojia Song}

China University of Petroleum, Beijing

\section{Weiyu Song}

China University of Petroleum, Beijing

\section{Yifeng Chen}

Central China Normal University

\section{Zhichao Wu}

Central China Normal University

\section{Ying Qin}

Central China Normal University

\section{Lei Jiao}

Central China Normal University

\section{Weiqing $\mathrm{Xu}$}

Central China Normal University

\section{Yu Wu}

Central China Normal University

\section{Jiajia Huang}

Central China Normal University

\section{Xiaoli Cai}

Central China Normal University

\section{Lirong Zheng}

Beijing Synchrotron Radiation Facility, Institute of High Energy Physics, Chinese Academy of Sciences

\section{Liuyong $\mathrm{Hu}$}

Wuhan Institute of Technology

\section{Wenling Gu}

Central China Normal University

Chengzhou Zhu ( $\nabla$ czzhu@mail.ccnu.edu.cn )

Central China Normal University https://orcid.org/0000-0003-0679-7965 
Keywords:

Posted Date: February 16th, 2022

DOI: https://doi.org/10.21203/rs.3.rs-1265628/v1

License: (c) (1) This work is licensed under a Creative Commons Attribution 4.0 International License. Read Full License 


\section{Abstract}

Although great progress has been made in nanozymes, their large-scale application still remains a huge challenge due to their unsatisfactory catalytic performances. Featuring unique electronic structure and coordination environment, single atoms nanozymes provide great opportunities to vividly mimic the specific metal catalytic center of natural enzymes and achieve the superior enzyme-like activity. Herein, spin-state engineering of single-atom nanozymes was employed for the first time to enhance peroxidase (POD)-like activity. Pd nanoclusters (PdNC) were introduced to rearrange spin electron occupation of Fe single atom (FeNC-PdNC), in which the electron-withdrawing properties of PdNC make the spin transiting of Fe single atoms from low spin to medium spin, facilitating the heterolysis process of $\mathrm{H} 2 \mathrm{O} 2$ and the desorption of $\mathrm{H} 2 \mathrm{O}$. As a result, the spin-rearranged FeNC-PdNC displays superior $\mathrm{H} 2 \mathrm{O} 2$ activating performance and kinetics to FeNC. Finally, FeNC-PdNC was applied to the immunosorbent assay for the colorimetric detection of prostate-specific antigen, achieving an ultralow detection limit of $0.38 \mathrm{pg} / \mathrm{mL}$. Our findings provide a new paradigm for designing advanced enzyme mimics and allow to gain a deep insight into the catalytic mechanism of nanozymes.

\section{Introduction}

Peroxidases (POD), a family of enzymes that catalytically oxidize certain compounds by virtue of peroxides $\left(\mathrm{H}_{2} \mathrm{O}_{2}\right.$ in most cases), plays a significant role in biological systems, such as detoxifying $\mathrm{H}_{2} \mathrm{O}_{2}$ in human bodies. ${ }^{1-2}$ More specifically, it can be also isolated from organisms and applied to biosensing, therapy, and environmental protection, etc. ${ }^{3-5}$ However, their large-scale industrialization remains an ongoing issue due to the disadvantages of high cost, difficult recovery and variability. ${ }^{6}$ To this end, nanozymes, a series of nanomaterials possessing enzyme-like activity, have been discovered to substitute natural enzymes with the merits of excellent stability, facile storage and easy production. 6-10 Nevertheless, one obstacle lying in nanozymes emanates from their low catalytic efficiency, which mainly arises from their insufficient intrinsic activity. ${ }^{7}$ Overcoming these limitations to acquire highly active PODlike nanozymes is, therefore, a subject of interest and priority.

Compared with simulating functionally, mimicking natural enzymes from the perspective of biological structure may provide a more reasonable strategy to achieve high catalytic performance. ${ }^{11-14}$ Specifically, the well-defined electronic and geometric structures of single-atom nanozymes render them appealing alternatives to natural enzymes by mimicking their highly evolved catalytic center at the atomic scale. ${ }^{12,15-16}$ Given the similar heme structure of horseradish peroxidase (HRP), Fe single-atom nanozymes are promising to catalyze $\mathrm{H}_{2} \mathrm{O}_{2}$ activation with remarkable activity. ${ }^{15,17-21}$ More importantly, the tunability of the local environment of single atoms is expected to optimize them towards enhanced performance. For example, heteroatoms, such as $\mathrm{B}, \mathrm{S}$ or $\mathrm{P}$, were introduced as a "modulator" to relieve the energy barrier during $\mathrm{H}_{2} \mathrm{O}_{2}$ reduction procedures. ${ }^{11,22-23}$ One $\mathrm{N}$ atom was adhered to the Fe single atom as the axial ligand $\left(\mathrm{FeN}_{5}\right)$ to emulate the penta-coordinated heme systems, which shows enhanced PODlike performance compared to the $\mathrm{FeN}_{4}$ counterpart. ${ }^{14}$ Furthermore, according to the frontier molecular 
orbital theory in molecular chemistry, the catalysis process is intensively interrelated to the electron donation and back-donation steps between the reactants/intermediates and metal center. ${ }^{24-25}$ During the reaction process with multistep intermediates production, the number of unpaired electrons cannot be always conserved, leading to the spin-related electron transfer and thus the $e_{g}$ orbital occupation and spintronic configuration sensitive reaction kinetics and thermodynamics. ${ }^{26}$ Inspired by this, the efforts in tuning the spin state of metal atoms in single-atom nanozymes are greatly expected to achieve a further breakthrough in the POD-like activity, achieving the efficient activation of $\mathrm{H}_{2} \mathrm{O}_{2}$ and revealing the underlying relationships between spin state and performance. To our best knowledge, spin-related design towards nanozymes has not been reported before.

In this work, spin-dependent FeNC single-atom nanozymes were proposed for the first time for POD mimicking by employing Pd nanoclusters $\left(\mathrm{Pd}_{\mathrm{NC}}\right.$ ) as a "modulator" (named as FeNC-Pd $\mathrm{NC}_{\mathrm{NC}}$ ). In virtue of the synergistic effect between $\mathrm{Pd}_{\mathrm{NC}}$ and $\mathrm{Fe}$ single atoms, $\mathrm{FeNC}-\mathrm{Pd}_{\mathrm{NC}}$ shows outstanding POD-like behavior in comparison to FeNC. The exploration of spin configurations of Fe single atoms manifests that the presence of $\mathrm{Pd}_{\mathrm{NC}}$ endows Fe single atoms with significantly improved content of medium spin (MS) $\mathrm{Fe}(\mathrm{II})$, which is proved to be more active than low spin (LS) Fe(II) by weakening the binding strength towards oxygen-contained intermediate, accelerating $\mathrm{H}_{2} \mathrm{O}_{2}$ activation to $\mathrm{H}_{2} \mathrm{O}$ along low energy trajectories (heterolytic path). The spin-dependent $\mathrm{H}_{2} \mathrm{O}_{2}$ reduction opens an avenue for controllable POD mimicking and introduces a feasible approach to significantly improve the efficiency of such reactions.

\section{Results And Discussion}

FeNC-Pd $\mathrm{NC}_{\mathrm{NC}}$ is prepared via the dual-confinement effect of the metal-organic frame (MOF), which is displayed in Figure 1a. FeNC with abundant Fe single atoms were prepared by pyrolysis Fe-doped Zeolitic imidazolate frameworks (ZIF-8) (Figure S1), then $\mathrm{Pd}_{\mathrm{NC}}$ were incorporated into FeNC through micropore adsorption strategy. ${ }^{27}$ Specifically, $\mathrm{Pd}^{2+}$ first interacts with carbon skeleton and subsequently be in-situ reduced by $\mathrm{H}_{2}$ to form $\mathrm{Pd}_{\mathrm{NC}}$. Notably, the rich metal nodes of ZIF-8 and the pores after annealing act as two "claws", achieving the anchoring both of Fe single atoms and $\mathrm{Pd}_{\mathrm{NC}} \cdot{ }^{28}$ For comparison, $\mathrm{NC}-\mathrm{Pd}_{\mathrm{NC}}$ was also prepared using ZIF-8 without Fe elements as the precursor to explore the role of $\mathrm{Pd}_{\mathrm{NC}}$. Scanning electron microscope (SEM) and transmission electron microscopy (TEM) images of FeNC-Pd ${ }_{N C}$ display their dodecahedral shape inherited from ZIF-8 (Figures 1b and S2). High-angle annular dark-field scanning TEM (HAADF-STEM) image confirms that the high-density and uniform $\mathrm{Pd}_{N C}$ are confined in the carbon structures with an average size of $2.13 \mathrm{~nm}$ (Figure 1c). As shown in Figure S3, all the as-prepared nanozymes possess both micropores and mesopores. Further observation finds that there exists clear shrinking of pore distributions around $2 \mathrm{~nm}$ after the integration of $\mathrm{Pd}_{\mathrm{NC}}\left(\mathrm{FeNC}_{\mathrm{Pd}} \mathrm{d}_{\mathrm{NC}}\right)$ in comparison to FeNC (inset in Figure S3b), manifesting the pivotal role of the micropore structures for FeNC in confining the growth of $\mathrm{Pd}_{\mathrm{NC}}$. The relatively decreased Brunauer-Emmett-Teller (BET) area from 554.86 $\mathrm{m}^{2} / \mathrm{g}$ (FeNC) to $547.03 \mathrm{~m}^{2} / \mathrm{g}\left(\mathrm{FeNC}_{\mathrm{N}} \mathrm{d}_{\mathrm{NC}}\right)$ proves this viewpoint (Table S1). Meanwhile, the rich surface area for all as-synthesized nanozymes also guarantees the accessibility of active sites for reactants. ${ }^{29}$ 
The aberration-corrected HAADF-STEM (AC-HAADF-STEM) image in Figure 1d reveals that numerous Fe single atoms surround $\mathrm{Pd}_{\mathrm{NC}}$, which may enhance the interactions between them. The enlarged $\mathrm{AC}$ HAADF-STEM and corresponding energy dispersive spectroscopy (EDS) mapping confirm the presence of uniformly distributed $\mathrm{Pd}$ species in $\mathrm{Pd}_{\mathrm{NC}}$ with the encircling of $\mathrm{Fe}$ species (Figure S4). As shown in Figure S5, the Pd(111) characteristic peaks are observed in the X-ray diffraction (XRD) patterns both for FeNC$\mathrm{Pd}_{\mathrm{NC}}$ and $\mathrm{NC}-\mathrm{Pd}_{\mathrm{NC}}$ compared to FeNC, confirming the successful introduction of $\mathrm{Pd}_{\mathrm{NC}}$. Inductively coupled plasma optical emission spectrometry (ICP-OES) analysis determines that Pd and Fe contents in FeNC-Pd $\mathrm{NC}_{\mathrm{NC}}$ are $\sim 4 \mathrm{wt} \%$ and $\sim 0.36 \mathrm{wt} \%$, respectively, consistent with feeding contents. As outlined in Figure S6, all nanozymes exhibit rich disorientated graphene structures with a high-intensity ratio of $D$ band and $G$ band $\left(I_{D} / I_{G}\right)$ from the Raman spectra results. ${ }^{30}$

To gain insight into the chemical state of various elements in nanozymes, X-ray photoelectron spectroscopy (XPS) analysis was carried out. The C 1s spectra shown in Figure S7a exhibit four peaks, associated with $\mathrm{sp}^{2}-\mathrm{C}, \mathrm{sp}^{3}-\mathrm{C}, \mathrm{C}-\mathrm{N}_{\mathrm{x}}$, and $\mathrm{C}=\mathrm{O} / \mathrm{C}-\mathrm{O} .{ }^{31}$ The $\mathrm{N} 1 \mathrm{~s}$ spectra can be deconvoluted into four peaks at $398.5,399.5,400.4$, and $401.1 \mathrm{eV}$, which are attributed to pyridinic $\mathrm{N}$, FeN, pyrrolic N, and graphitic $\mathrm{N}$ (Figure S7b). ${ }^{32}$ The high ratios of FeN contents among all N species for FeNC-Pd $\mathrm{NC}_{\mathrm{NC}}(23.1 \%)$ and FeNC $(21.6 \%)$ illustrate their rich Fe single atoms species (Table S2). Especially, the Fe 2p spectrum for FeNC$\mathrm{Pd}_{\mathrm{NC}}$ displays an obvious peak shift $(0.3 \mathrm{eV})$ towards higher binding energy with the integration of $\mathrm{Pd}_{\mathrm{NC}}$ and Pd shows the decreased binding energy compared to NC-Pd $\mathrm{NC}_{\mathrm{NC}}$ (Figures $1 \mathrm{e}$ and S8). This result implies the electron transfer from Fe single atoms to $\mathrm{Pd}_{\mathrm{NC}}$, resulting from electronegativity discrepancy between $\mathrm{Pd}$ and $\mathrm{Fe}^{2+} .{ }^{33}$ Specifically, the decreased electron density of the Fe center in $\mathrm{FeNC}-\mathrm{Pd} \mathrm{NC}_{\mathrm{NC}}$ may significantly regulate the catalytic behavior.

To further elucidate the change of local chemical configuration of Fe species after the introduction of $\mathrm{Pd}_{\mathrm{NC}}$, Fe K-edge X-ray absorption fine structure (XAFS) was then performed. As depicted in Figure 1f, the Fe K-edge extended-XAFS (EXAFS) Fourier-transformed magnitudes both of FeNC-Pd $\mathrm{NC}_{\mathrm{NC}}$ and FeNC show major peaks at $\sim 1.4 \AA$, consistent well with that in iron-porphyrin $(\mathrm{Fe} P \mathrm{Pc}$ ) and attributed to the backscattering between $\mathrm{Fe}$ and $\mathrm{N}$ atoms. ${ }^{11}$ The FeN coordination number values based on the quantitative EXAFS fitting analysis are 4.1 and 5.1 within FeNC-Pd ${ }_{\mathrm{NC}}$ and FeNC, respectively (Table S3). Note that the additional atoms compared to traditional $\mathrm{FeN}_{4}$ moieties may be derived from the $\mathrm{O}$ species in the measuring environment, which can be easily removed during the catalytic process. ${ }^{34}$ Then, X-ray absorption near edges structure (XANES) spectra were applied to identify the valence state of Fe species. As displayed in Figure 1 $\mathbf{g}$, the absorption edge of Fe K-edge XANES curves for FeNC-Pd $\mathrm{NC}_{\mathrm{NC}}$ and FeNC is close to Fe Pc, indicating the atomically dispersed Fe with the valence state near $+2 .{ }^{35}$ Intriguingly, the valence state of $\mathrm{FeNC}-\mathrm{Pd} \mathrm{NC}_{\mathrm{NC}}$ appears to be more positive than that of $\mathrm{FeNC}$, illustrating the decreased electron density and matching well with the XPS results. ${ }^{36}$

Due to the variation of coordination environment, $\mathrm{N}$ coordinated $\mathrm{Fe}$ (II) tends to exhibit multiple states, which display different forms of spin ( $L S t_{2 g} 6 e_{g} 0$, MS $t_{2 g} 5 e_{g} 1$ and high spin (HS) $t_{2 g} 3 e_{g} 3$ ). ${ }^{37}$ As for LS 
Fe (II), each Fe atom splits to empty higher $e_{g}$ and fully occupied lower $t_{2 g}$ orbits (Figure $\left.2 a\right)$. When it transforms into MS Fe (II), one electron from $t_{2 g}$ orbit will hop to $e_{g}$ orbit, producing unpaired electrons on the half-filled $\mathrm{e}_{\mathrm{g}}$ orbit. During the $\mathrm{H}_{2} \mathrm{O}_{2}$ reduction procedure, the hybridization between the $2 \mathrm{p}$ orbit of $\mathrm{O}$ for $\mathrm{H}_{2} \mathrm{O}_{2}$ /intermediates (including $\mathrm{OH}^{*}, \mathrm{O}^{*}$ and $\mathrm{H}_{2} \mathrm{O} *$ ) and $3 \mathrm{~d}$ orbits of Fe sites will take place, making the $3 \mathrm{~d}$ electronic state act as spin-dependent gates to regulate electron transfer and orbital interaction in the catalytic reaction. ${ }^{24}$ Importantly, the charge redistribution primarily dominates the electronic configuration of $\mathrm{Fe}(\mathrm{II})$, i.e., spin state, and thus regulates the catalytic activity. To confirm this conception, the $\mathrm{H}_{2} \mathrm{O}_{2}$ reduction process in both LS and MS Fe(II) is theoretically predicted through density functional theory (DFT) calculations. It is reported that Fe-based catalysts usually bind oxygen too strongly, circumscribing the proton-electron transfer and limiting the catalytic activity. Due to the scaling relationships of different intermediates, $0^{*}$ is chosen as a model to discuss its interaction with Fe sites, which was investigated based on the density of states (DOS) and crystal orbital Hamilton population (COHP) analysis. ${ }^{38}$ Besides, due to $\mathrm{Fe}-\mathrm{d}_{\mathrm{xy}}$ and orbits of the metal site cannot mix with the ${ }^{*} \mathrm{O}$ orbitals based on symmetry conservation, the interaction between them and the orbits of the adsorbed $0^{*}$ is negligible. ${ }^{39}$ As shown in Figure $\mathbf{2} \mathbf{a}$, the interaction between Fe center and adsorbed $\mathrm{O}^{*}$ is changed along with the spin state of $\mathrm{Fe}(\mathrm{II})$. Specifically, half-filled orbit of MS Fe (II) can accept fewer valence electrons from *0 with the generation of one $s^{*}$ orbit, which is more favorable for the desorption of $\mathrm{H}_{2} \mathrm{O}_{2}$ /intermediates to trigger a catalytic cycle (Figure S9). In this case, it is assumed that if local microenvironment disturbance is exerted to modulate the electronic hopping and occupation of $3 \mathrm{~d}$ orbit, spin reconfiguration can be achieved, further realizing the regulation of enzyme-like performance.

Subsequently, the electronic spin information of Fe single atoms before and after integrating with $\mathrm{Pd}_{\mathrm{NC}}$ was investigated. $\mathrm{FeN}_{4}$ embed within single-layer graphene was used to simulate the FeNC, while the FeNC coupling with five-layer Pd (111) facets was used to simulate FeNC-Pd $\mathrm{NC}_{\mathrm{NC}}$. As shown in Figure $2 b$, the projected DOS diagrams reveal that the distance between the d-band center of spin up and spin down $\left(\left|\varepsilon_{\mathrm{d} \text {-up }}-\varepsilon_{\mathrm{d} \text {-down }}\right|\right)$ increases with the introduction of $\mathrm{Pd}_{\mathrm{NC}}$, indicating the higher spin state of Fe center. The integral projected density of states (IPDOS) analysis of splitting Fe 3d orbits displays that this increasing spin state mainly originates from the increasing Fe contributions with the electron loss in $d_{x z}$ $\mathrm{d}_{\mathrm{yz}}$ orbits (Figure $2 \mathrm{c}$ ), which can be also visualized by the spin density diagram (Figure S10). Especially, the highest occupied molecular orbital (HOMO) for Fe(II) in FeNC-Pd $d_{N C}$ shows one unique orbit perpendicular to the plane within the introduction of $\mathrm{Pd}_{\mathrm{NC}}$, while such orbit was absent in parent $\mathrm{FeNC}$ (Figure $\mathbf{2 c}$ below). To verify this kind of spin redistribution experimentally, zero-field cooling temperaturedependent (ZFC-T) magnetic susceptibility $\left(\chi_{m}\right)$ was carried out. As shown in Figure $2 \mathbf{d}$, the $\chi_{m}$ derived from the magnetizations follows by Curie-Weiss law both for FeNC and FeNC-Pd $\mathrm{NC}_{\mathrm{NC}}$. Then the total

effective magnetic moment $\left(\mu_{\text {eff }}\right)$ can be obtained according to the equation:

$$
2.828 \sqrt{\chi_{\mathrm{m}} \mathrm{T}}=\mu_{\text {eff }}=\sqrt{\mathrm{n}(\mathrm{n}+2)}
$$

calculate the number of single spin electrons $\left(n_{s}\right) \cdot{ }^{40}$ Especially, the $n_{s}$ of $F e(I I)$ increases from 0 (FeNC) to $2\left(\mathrm{FeNC}-\mathrm{Pd}_{\mathrm{NC}}\right)$, which testifies the increasing spin state and is in accord with the DFT results. For 
quantification, Mössbauer spectroscopy analysis, a powerful tool for the identification of the oxidation, coordination structure and the spin polarization configuration of Fe was conducted. Three different doublets (D1-D3) can be fitted for FeNC-Pd ${ }_{\mathrm{NC}}$ and FeNC, corresponding to LS Fe(II), MS Fe(II) and HS $\mathrm{Fe}(\mathrm{II})$, respectively. ${ }^{41}$ Significantly, FeNC-Pd $\mathrm{NC}_{\mathrm{NC}}$ possesses higher content of MS Fe(II) (33.4\%) compared to FeNC (17.1\%) (Figure 2e and Table S4). By this token, the strong interactions between Fe single atoms and $\mathrm{Pd}_{\mathrm{NC}}$ effectively reshape the electronic structure of $\mathrm{Fe}$, and achieve the $\mathrm{Fe} 3 \mathrm{~d}$ electron-spin configuration transition from LS to MS. It is reckoned that this intrinsically spin-state transition may endow $\mathrm{Fe}-\mathrm{N}-\mathrm{C} / \mathrm{Pd}_{\mathrm{NC}}$ with high catalytic activity according to the DFT prediction.

Following the structural characterization, the POD-like catalytic activity of FeNC-Pd $\mathrm{NC}_{\mathrm{NC}}$ was quantitively assessed using a chromogenic biochemical reaction of 3,3',5,5'-tetramethylbenzidine (TMB), which can be oxidized into ox-TMB with blue color by $\mathrm{H}_{2} \mathrm{O}_{2} \cdot{ }^{42-43}$ As expected, $\mathrm{FeNC}-\mathrm{Pd}_{\mathrm{NC}}$ exhibits high catalytic activity with strong characteristic absorbance of ox-TMB at $652 \mathrm{~nm}$, approximately twice as high as that of FeNC (Figure 3a). Moreover, specific activity (SA) was investigated to quantitatively determine catalytic performance. As shown in Figure 3b, the SA value of FeNC-Pd $\mathrm{NC}_{\mathrm{NC}}$ is $95.68 \mathrm{U} \cdot \mathrm{mg}^{-1}$, more than 3-fold higher than that of FeNC (30.27 U. $\left.\mathrm{mg}^{-1}\right)$. Meanwhile, the potassium thiocyanate (KSCN) poisoning experiment shows that the injection of $\mathrm{SCN}^{-}$prominently impairs the POD-like performance of FeNC-Pd $\mathrm{NC}_{\mathrm{N}}$, confirming that Fe single atoms are the main active centers for the POD-like property (Figure S11). ${ }^{13}$ Thus, all of the above results illustrate that Fe single atoms play a critical role in POD-like activity and the existence of $\mathrm{Pd}_{\mathrm{NC}}$ can significantly boost its activity, despite the sluggish kinetics of $\mathrm{H}_{2} \mathrm{O}_{2}$ reduction at $\mathrm{Pd}_{\mathrm{NC}}$. Furthermore, the unsatisfactory specificity of inorganic nanostructures has been a major constraint on their development. ${ }^{44}$ Therefore, the activity of oxidase (OXD)-like, the major interference reaction of PODlike was also evaluated (Figures $3 c$ and S12). Unlike POD-like activity, the OXD-like activity of FeNC-Pd $\mathrm{NC}_{\mathrm{N}}$ shows negligible enhancement after the integration of $\mathrm{Pd}_{\mathrm{NC}}$, indicating that the improved performance is mainly derived from elevated POD-like activity, rather than OXD-like one. More importantly, by comparing the activities of FeNC-Pd $d_{N C}, F e N C$ and NC-Pd $d_{N C}$, we find that the POD-like performance of FeNC-Pd $d_{N C}$ is much higher than that superimposed value of FeNC and NC-Pd $\mathrm{NC}_{\mathrm{NC}}$, powerfully confirming the key role of the synergistic effect between $\mathrm{Pd}_{\mathrm{NC}}$ and Fe single atoms towards the catalytic performance. Furthermore, to evaluate the affinity of POD mimics toward the substrate, Michaelis constant $\left(\mathrm{K}_{\mathrm{m}}\right)$ and maximum reaction rate $\left(\mathrm{V}_{\text {max }}\right)$ were obtained from Michaelis-Menten curves (Figures $3 \mathrm{~d}$ and $\left.\mathrm{S} 13\right)$ ). The $\mathrm{V}_{\max }$ of FeNC-Pd $\mathrm{NC}_{\mathrm{NC}}$ towards $\mathrm{H}_{2} \mathrm{O}_{2}$ is $10.93 \mu \mathrm{M} / \mathrm{s}, 1.99$ times higher than that of FeNC $(5.49 \mu \mathrm{M} / \mathrm{s})$. Similarly, FeNC-Pd $\mathrm{NC}_{\mathrm{NC}}$ also exhibits a higher $\mathrm{V}_{\mathrm{max}}$ value than FeNC towards TMB (Table S5). Besides, the $\mathrm{K}_{\mathrm{m}}$ of FeNC-Pd $d_{N C}$ and FeNC towards $\mathrm{H}_{2} \mathrm{O}_{2}$ is $9.58 \mathrm{mM}$ and $14.02 \mathrm{mM}$, respectively. The lower $\mathrm{K}_{\mathrm{m}}$ of FeNC-Pd demonstrates its higher affinity towards $\mathrm{H}_{2} \mathrm{O}_{2}$. All of these results display that $\mathrm{FeNC}-\mathrm{Pd}_{\mathrm{NC}}$ possesses a higher affinity towards substrates, making it better POD-like catalytic performance than FeNC. Similar to natural HRP, both FeNC-Pd $\mathrm{NC}_{\mathrm{N}}$ and FeNC exhibits high pH-dependent properties (Figure S14). Moreover, they possess a much higher temperature tolerance than HRP. The as-prepared nanozymes maintain their POD-like activity after the treatment of strong acidic and alkaline solution compared to HRP, illustrating 
that they can perfectly surmount the disadvantage of the inferior stability of natural enzymes (Figure S15).

To understand the potential catalytic intermediates, electron paramagnetic resonance (EPR) spectroscopy was then performed by using 5,5-dimethyl-1-pyrroline $\mathrm{N}$-oxide (DMPO) as $\cdot \mathrm{OH}$ trapping agent. Unlike the $\mathrm{Fe}^{2+} / \mathrm{H}_{2} \mathrm{O}_{2}$ system, no signal of $\cdot \mathrm{OH}$ was detected in all nanozymes $/ \mathrm{H}_{2} \mathrm{O}_{2}$, which is similar to $\mathrm{HRP} / \mathrm{H}_{2} \mathrm{O}_{2}$ (Figure $3 e$ ). What's more, the existence of $\bullet \mathrm{OH}$ radical scavengers (t-butyl alcohol, TBA) has relatively smaller effects on the activity of FeNC-Pd $\mathrm{NC}_{\mathrm{NC}}$ and FeNC, indicating that $\bullet \mathrm{OH}$ is not the critical active intermediate during the catalytic reaction (Figure S16). Inspired by the catalytic mechanism of natural HRP, Fe(IV) $=0$ intermediate is considered as the active intermediate. ${ }^{45-46}$ Thus, low-temperature (77 K) EPR experiment was performed to detect the signal of $\mathrm{Fe}(\mathrm{IV})=0$. As expected, obvious rhombic signals at $\mathrm{g}=2$ are detected after reaction with excess $\mathrm{H}_{2} \mathrm{O}_{2}$ both for FeNC-Pd $\mathrm{NC}_{\mathrm{NC}}$ and $\mathrm{FeNC}$, suggesting the formation of $\mathrm{Fe}(\mathrm{IV})=0$ species during the reaction process (Figure $3 \mathrm{f}$ ). ${ }^{47}$ Meanwhile, the intensity of FeNC is much smaller than that of FeNC-Pd ${ }_{N C}$. Taking the heme-like structure of FeNC similar to the natural HRP into account, we speculate that $\mathrm{Fe}(\mathrm{IV})=0$ species might be the essential active intermediate via the bound ROS pathway to oxide TMB, while the presence of $\mathrm{Pd}_{\mathrm{NC}}$ is expected to enhance the production of $\mathrm{Fe}(\mathrm{IV})=0$ species.

As discussed above, the integration of $\mathrm{Pd}_{\mathrm{NC}}$ achieves the spin transition of $\mathrm{Fe}(\mathrm{II})$ and thus enhances the POD-like activity of Fe single atoms. To theoretically explain the effect of spin state on the POD-like activity, DFT calculations were performed. As shown in Figures 4 a and S17, the $\mathrm{H}_{2} \mathrm{O}_{2}$ heterolytic dissociation route is energetically more favorable for FeNC and FeNC-Pd $d_{N C}$. Especially, the $F e(I V)=0$ intermediate, which plays a critical role in oxidizing TMB similar to natural HRP, was produced by the dehydration adsorbed $\mathrm{H}_{2} \mathrm{O}_{2}\left(\mathrm{H}_{2} \mathrm{O}_{2} \rightarrow \mathrm{O}^{*}+\mathrm{H}_{2} \mathrm{O}\right) .{ }^{48}$ As presented in Figure $4 \mathrm{~b}$, the $\mathrm{H}_{2} \mathrm{O}_{2}$ dehydration process is thermodynamically energy uphill with the energy barrier of $0.75 \mathrm{eV}$ on FeNC. After the integration of $\mathrm{Pd}_{\mathrm{NC}}$, this energy barrier is largely decreased to $0.36 \mathrm{eV}$ on FeNC-Pd $\mathrm{N}_{\mathrm{NC}}$, promoting the formation of $\mathrm{Fe}(\mathrm{IV})=0$ intermediate sites. As discussed above, the spin state of $\mathrm{Fe}(\mathrm{II})$ fluctuates the interaction between Fe sites and ${ }^{*} \mathrm{O}$, which may further improve its oxidation capacity towards $T M B$. Then, the effect of $\mathrm{Pd}_{\mathrm{NC}}$ integration on TMB oxidization was evaluated. The oxidation of TMB was proceeded through the $\mathrm{N}-\mathrm{H}$ bond cleavage of $\mathrm{TMB}$ and transformation $\mathrm{H}$ to $\mathrm{Fe}(\mathrm{IV})=0$ with the electron transfer from the substrate to nanozymes. As a result, the dissociation energy of TMB on $\mathrm{Fe}(\mathrm{IV})=0$ decreases from $0.55 \mathrm{eV}$ (FeNC) to $0.24 \mathrm{eV}\left(\mathrm{FeNC}_{\mathrm{Pd}} \mathrm{NC}\right)$, rendering it more favorable to oxide TMB (Figure 4c).

As depicted in Figure 4a, the $\mathrm{H}_{2} \mathrm{O}$ * desorption on FeNC is the rate-determining step (RDS) with the energy barrier of $0.28 \mathrm{eV}$ due to the strong binding between Fe single atoms and oxygen-containing species. Encouragingly, this step becomes exothermic for FeNC-Pd $\mathrm{NC}_{\mathrm{NC}}$. To get a better understanding, the orbital interactions between $\mathrm{H}_{2} \mathrm{O}^{*}$ and Fe sites were explored. The COHP and DOS analysis show that the bonding contribution for FeNC-Pd $\mathrm{NC}_{\mathrm{NC}}$ shifts towards lower energy while the antibonding contribution shifts toward higher energy, which can weaken the $\mathrm{Fe}-\mathrm{O}$ bond strength in $\mathrm{H}_{2} \mathrm{O}^{*}$ adsorption configuration 
(Figures $4 \mathrm{~d}$ and Figure S18). Furthermore, an axial $\sigma^{\star}$ antibonding molecular orbital can be observed for $\mathrm{H}_{2} \mathrm{O}^{*}$ on FeNC-Pd $\mathrm{NC}_{\mathrm{NC}}$ due to the higher electron occupation, while the antibonding contribution mainly comprises from $\mathrm{p}^{*}$ antibonding orbitals for FeNC. This unique $\sigma^{*}$ antibonding contributes to the timely $\mathrm{H}_{2} \mathrm{O}$ desorption on FeNC-Pd $\mathrm{d}_{\mathrm{NC}}$. The above DFT results support that FeNC-Pd $\mathrm{d}_{\mathrm{NC}}$ with more ratio of MS $\mathrm{Fe}(\mathrm{II})$ exhibits high-efficiency POD-like performance.

In a proof-of-principle demonstration, FeNC-Pd $\mathrm{NC}_{\mathrm{NC}}$ was applied as labels to colorimetric enzyme-linked immunosorbent assay (ELISA) and prostate-specific antigen (PSA, a biomarker of prostate cancer) was chosen as a model analyte (Figure 5a). ${ }^{49-50}$ For comparison, HRP was also used to establish the HRPELISA. ${ }^{51}$ Typically, the surface of FeNC-Pd $\mathrm{NC}_{\mathrm{N}}$ is positively charged, which can conjugate with the labeling antibody of PSA $\left(\mathrm{Ab}_{2}\right)$ as signal tags through electrostatic interaction (Figure S19). Confocal laser scanning microscope was used to prove the conjunction between FeNC-Pd $\mathrm{NC}_{\mathrm{NC}}$ and $A \mathrm{~b}_{2}$ with the label of rhodamine $B$. The red fluorescence emission demonstrates the successful immobilization of $A b_{2}$ on FeNC-Pd $_{\mathrm{NC}}$ (Figure S20). The absorption spectrum and corresponding calibration curves were obtained, where FeNC-Pd $\mathrm{NC}_{\mathrm{NC}}$ ELISA displays a wide detection range of $1-2000 \mathrm{pg} / \mathrm{mL}$ with the lower variable coefficient of $0.18 \%-5.18 \%$ (Figures $5 \mathrm{~b}$ and S21). The limit of detection (LOD, defined by the 3SD method) is determined to be $0.38 \mathrm{pg} / \mathrm{mL}$. In contrast, the LOD of HRP ELISA is calculated to be 5.38 $\mathrm{pg} / \mathrm{mL}$ with a relatively narrow detection range between $10-1000 \mathrm{pg} / \mathrm{mL}$ (Figure S22). Also, the sensitivity towards PSA is much higher than those of previous reports, suggesting that $\mathrm{FeNC}-\mathrm{Pd}_{\mathrm{NC}}$ hold great promise in immunoassay. (Table S6) ${ }^{13,49,52-54}$

For the specificity, obvious characteristic intensity can be observed for PSA, while there are almost no changes for a series of other biomarkers or metal ions compared to blank samples (Figure $5 \mathrm{c}$ ). In the presence of all of the above interferents, the FeNC-Pd $\mathrm{NC}_{\mathrm{N}}$ ELISA still maintains the signal values towards PSA, indicating its good selectivity and anti-interference ability. Furthermore, FeNC-Pd $d_{N C}$ ELISA also exhibits outstanding repeatability with the low calculated relative standard deviation (RSD) of $4.23 \%$ through five independent tests (Figure S23). Similarly, the stability was also carried out in the same way in ten days, where the RSD is calculated as low as 3.29\% (Figure S24). By taking advantage of high activity, selectivity and stability, FeNC-Pd $\mathrm{NC}_{\mathrm{NC}}$ ELISA was used to detect PSA in the real serum sample. As expected, the results of FeNC-Pd $\mathrm{NC}_{\mathrm{NC}}$ ELISA is well consistent with the chemiluminescence analysis results, indicating the remarkable feasibility and accuracy in the practical application for the proposed assay (Figure 5d). Thus, FeNC-Pd $\mathrm{NC}_{\mathrm{N}}$ ELISA holds great promise in clinical diagnosis and can be developed for the detection of other biomarkers.

\section{Conclusion}

In this work, spin-dependent Fe single-atom nanozymes were proposed to achieve enhanced POD-like activity. Experimental and theoretical calculations reveal that the electron-withdrawing effect of $\mathrm{Pd}_{\mathrm{NC}}$ induces the spin transition of Fe single atom from the LS to MS with the increased electron occupation 
in orbit. More interestingly, charge transfer and orbital interaction between the Fe sites and the intermediates display spin-dependent features, where the higher electron occupation is beneficial to the generation of the essential $\mathrm{Fe}(\mathrm{IV})=\mathrm{O}$ intermediates and promotes the timely desorption of $\mathrm{H}_{2} \mathrm{O}$. Thanks to the superior catalytic activity, $\mathrm{FeNC}_{\mathrm{Nd}} \mathrm{d}_{\mathrm{NC}}$ has been used to construct ELISA for sensitive detection of PSA, which exhibits satisfactory selectivity and sensitivity. Together, this work offers new insight into the design and development of enzyme-like catalysts. More importantly, we believe that spin-related catalyst design emerges as an important strategy and helps to get a comprehensive understanding of the catalytic nature of nanozymes.

\section{Methods}

Synthesis of FeNC. FeNC was synthesized referring to the reference. ${ }^{55}$ During the synthesis, $3.94 \mathrm{~g} 2-$ methylimidazole was dissolved in $300 \mathrm{~mL}$ methanol under vigorous stirring, followed by adding $300 \mathrm{~mL}$

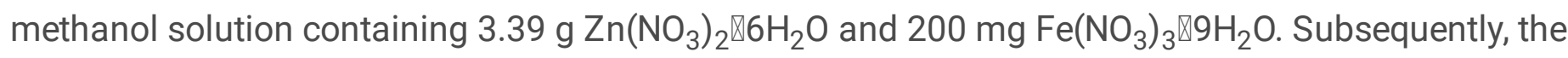
solution was heated at $60^{\circ} \mathrm{C}$ for $24 \mathrm{~h}$. The precipitant was centrifuged followed by thorough washing with ethanol repeatedly to remove unreacted linkers and dried at $60^{\circ} \mathrm{C}$ in a vacuum oven to obtain $\mathrm{Fe} / \mathrm{ZIF}-8$ crystals. Then FeNC was prepared by pyrolyzing Fe/ZIF-8 crystals at the temperature of $900{ }^{\circ} \mathrm{C}$ with a ramp rate of $5{ }^{\circ} \mathrm{C} / \mathrm{min}$ under $\mathrm{N}_{2}$ flow for $2 \mathrm{~h}$ in a tube furnace. No additional acidic leaching was carried out.

Synthesis of FeNC-Pd $\mathrm{NC}_{\mathrm{NC}}$. To obtain FeNC-Pd $\mathrm{NC}_{\mathrm{NC}}, 40 \mathrm{mg}$ FeNC was dispersed in $50 \mathrm{~mL}$ water, vigorously sonicating for $10 \mathrm{~min}$. Subsequently, $0.76 \mathrm{~mL} \mathrm{Na}{ }_{2} \mathrm{PdCl}_{4}$ solution $(19.7 \mathrm{mM})$ was dropwise added slowly into the above suspension under vigorous stirring. To sufficiently impregnate, the solution was stirred continuously for 24 hours, followed by washingwith pure water several times and dried at $60{ }^{\circ} \mathrm{C}$ in a vacuum oven. Then the adsorbed Pd precursor was reduced at $5 \% \mathrm{H}_{2}$ with a ramp rate of $5{ }^{\circ} \mathrm{C} / \mathrm{min}$ to 400 ${ }^{\circ} \mathrm{C}$, maintaining $2 \mathrm{~h}$ to obtain the final FeNC-Pd $\mathrm{NC}_{\mathrm{NC}}$. The mass fraction of Pd precursor accounts for $4 \%$ of the total precursors in FeNC-Pd ${ }_{\mathrm{NC}}$.

Synthesis of NC-Pd$d_{N C}$. The procedure of synthesis of $\mathrm{N}-\mathrm{C} / \mathrm{Pd} \mathrm{d}_{\mathrm{NC}}$ was similar to that of $\mathrm{NC}-\mathrm{Pd} \mathrm{d}_{\mathrm{NC}}$, except for the addition of Fe precursor during the preparation of ZIF-8 crystals.

\section{Evaluation of the peroxidase-like activity.}

The peroxidase-like property was confirmed by the color change of TMB, which is indicated by the absorbance at $652 \mathrm{~nm}$. Typically, $10 \mu \mathrm{L} 1 \mathrm{mg} \mathrm{mL}^{-1} \mathrm{FeNC}-\mathrm{Pd}_{\mathrm{NC}}$, FeNC or NC-Pd $\mathrm{NC}_{\mathrm{NC}}$ were introduced into the HAc-NaAc buffer $(0.1 \mathrm{M}, \mathrm{pH} 3.5,150 \mu \mathrm{L})$ containing $\mathrm{H}_{2} \mathrm{O}_{2}(100 \mathrm{mM}, 100 \mu \mathrm{L})$ and TMB $(1 \mathrm{mM}, 50 \mu \mathrm{L})$. Then, the absorbance values of the reaction solution were obtained by a multimode reader after 5 min.

\section{Kinetics Assay}


The kinetic data were detected by the change in concentration of $\mathrm{H}_{2} \mathrm{O}_{2}$ or TMB. Typically, $10 \mu \mathrm{L}$ of FeNC$\mathrm{Pd}_{\mathrm{NC}}, \mathrm{FeNC}$ or $\mathrm{NC}-\mathrm{Pd}_{\mathrm{NC}}$ were added into the mixture including $50 \mu \mathrm{L}$ of TMB $(10 \mathrm{mM}), 150 \mu \mathrm{L}$ of HAc$\mathrm{NaAc}(0.1 \mathrm{M}, \mathrm{pH} 3.5)$ and different concentrations of $\mathrm{H}_{2} \mathrm{O}_{2}(100 \mu \mathrm{L})$. The kinetic data were calculated by a typical Michaelis-Menten curve as to $v=\mathrm{V}_{\max }[S] /\left(\mathrm{K}_{\mathrm{m}}+[\mathrm{S}]\right)$, where $\mathrm{v}$ is the initial velocity, $[\mathrm{S}]$ is the concentration of the substrate, $\mathrm{K}_{\mathrm{m}}$ is the Michaelis-Menten constant, and $\mathrm{V}_{\max }$ is the maximal reaction velocity.

\section{Specific activities measurements}

The specific activity (SA) of FeNC-Pd $\mathrm{NC}_{\mathrm{N}}$ and FeNC was confirmed by the color change of TMB, which is indicated by the absorbance at $652 \mathrm{~nm}$. Various concentrations of FeNC-Pd $\mathrm{NC}_{\mathrm{NC}}$ and FeNC were added into the mixture including $100 \mu \mathrm{L}$ of $\mathrm{H}_{2} \mathrm{O}_{2}(10 \mathrm{M}), 150 \mu \mathrm{L}$ of $\mathrm{HAc}-\mathrm{NaAc}(0.1 \mathrm{M}, \mathrm{pH} 3.5)$, and $50 \mu \mathrm{L}$ of TMB (100 $\mathrm{mM})$. The SA was calculated by the following equation:

$$
\mathrm{SA}=\frac{\mathrm{V} /(\varepsilon \times \mathrm{I}) \times(\Delta \mathrm{A} / \mathrm{At})}{\mathrm{m}}
$$

$V$ is the total volume of the reaction solution $(\mu \mathrm{L}) ; \varepsilon$ is the molar absorption coefficient of the colorimetric substrate, which is typically maximized at $39,000 \mathrm{M}^{-1} \mathrm{~cm}^{-1}$ at $652 \mathrm{~nm}$ for TMB; I is the path length of light traveling in the cuvette $(\mathrm{cm}) ; A$ is the absorbance after subtraction of the blank value, and $\Delta \mathrm{A} / \Delta \mathrm{t}$ is the initial rate of change in absorbance at $652 \mathrm{~nm} \mathrm{~min}{ }^{-1} ; \mathrm{m}$ is the nanozymes weight $(\mathrm{mg})$ of each assay.

\section{Data availability}

The authors declare that all data supporting the findings of this study are included within the article and its Supplementary Information, as well as from the corresponding author upon reasonable request.

\section{Declarations}

\section{Acknowledgments}

The authors gratefully acknowledge the financial support of the National Natural Science Foundation of China (nos. 22074049, 22004042 and 22104114), the Fundamental Research Funds for the Central Universities (nos. CCNU20QN007 and CCNU20TS013) and the Program of Introducing Talents of Discipline to Universities of China (111 program, B17019). We thank the 1W1B station in Beijing Synchrotron Radiation Facility (BSRF) for X-ray absorption spectroscopy measurements.

\section{Author contributions}

C.Z. directed this research. X.W. designed the experiments and wrote the paper. S.S. and W.S. conducted the theoretical calculations. Y.C., Z.W., Y.Q., W.X., Y.W., J.H., X.C. helped to perform the experiments and reviewed the manuscript. L.Z. carried out the X-ray absorption experiments and L.J. contributed to XAS 
data analysis. L.H. and and W.G. provided helpful discussions. X.W. and S.S. contributed equally to this work. All authors discussed and revised the manuscript.

\section{Competing interests}

The authors declare no competing interests.

\section{References}

1 Komkova, M. A., Karyakina, E. E. \& Karyakin, A. A. Catalytically Synthesized Prussian Blue Nanoparticles Defeating Natural Enzyme Peroxidase. J. Am. Chem. Soc.140, 11302-11307 (2018).

2 Cheng, Y. et al. An Intracellular $\mathrm{H}_{2} \mathrm{O}_{2}$-Responsive AlEgen for the Peroxidase-Mediated Selective Imaging and Inhibition of Inflammatory Cells. Angew. Chem. Int. Ed. 57, 3123-3127 (2018).

3 Liu, B., Sun, Z., Huang, P. J. \& Liu, J. Hydrogen Peroxide Displacing DNA from Nanoceria: Mechanism and Detection of Glucose in Serum. J. Am. Chem. Soc. 137, 1290-1295 (2015).

4 Xu, B. et al. A Single-Atom Nanozyme for Wound Disinfection Applications. Angew. Chem. Int. Ed. 58, 4911-4916 (2019).

5 Herget, K. et al. Haloperoxidase Mimicry by $\mathrm{CeO}_{2}-x$ Nanorods Combats Biofouling. Adv. Mater. 29, 1603823 (2017).

6 Liang, M. \& Yan, X. Nanozymes: From New Concepts, Mechanisms, and Standards to Applications. Acc. Chem. Res. 52, 2190-2200 (2019).

7 Xi, Z. et al. Nickel-Platinum Nanoparticles as Peroxidase Mimics with a Record High Catalytic Efficiency. J. Am. Chem. Soc. 143, 2660-2664 (2021).

8 Wang, Y. et al. Coordination Number Regulation of Molybdenum Single-Atom Nanozyme Peroxidaselike Specificity. Chem 7, 436-449 (2021).

9 Wu, J. et al. Nanomaterials with Enzyme-Like Characteristics (Nanozymes): Next-Generation Artificial Enzymes (II). Chem. Soc. Rev. 48, 1004-1076 (2019).

10 Gao, L. et al. Intrinsic Peroxidase-Like Activity of Ferromagnetic Nanoparticles. Nat. Nanotechnol. 2, 577-583 (2007).

$11 \mathrm{Ji}$, S. et al. Matching the Kinetics of Natural Enzymes with a Single-Atom Iron Nanozyme. Nat. Catal. 4, 407-417 (2021).

12 Huang, L. et al. Single-Atom Nanozymes. Sci. Adv. 5, 5490 (2019). 
13 Chen, Y. et al. Fe-N-C Single-Atom Catalyst Coupling with Pt Clusters Boosts Peroxidase-like Activity for Cascade-Amplified Colorimetric Immunoassay. Anal. Chem. 93, 12353-12359 (2021).

$14 \mathrm{Xu}, \mathrm{W}$. et al. Axial Ligand-Engineered Single-Atom Catalysts with Boosted Enzyme-Like Activity for Sensitive Immunoassay. Anal. Chem. 93, 12758-12766 (2021).

15 Jiao, L. et al. When Nanozymes Meet Single-Atom Catalysis. Angew. Chem. Int. Ed. 59, 2565-2576 (2020).

16 Zhang, H. et al. Bionic Design of Cytochrome c Oxidase-Like Single-Atom Nanozymes for Oxygen Reduction Reaction in Enzymatic Biofuel Cells. Nano Energy 83, 105798 (2021).

17 Wei, X. et al. Recent Advances in Synergistically Enhanced Single-Atomic Site Catalysts for Boosted Oxygen Reduction Reaction. Nano Energy 84, 105817 (2021).

18 Chen, Y. et al. Thermal Atomization of Platinum Nanoparticles into Single Atoms: An Effective Strategy for Engineering High-Performance Nanozymes. J. Am. Chem. Soc. 143, 18643-18651 (2021).

19 Zhu, Y. et al. Stimuli-Responsive Manganese Single-Atom Nanozyme for Tumor Therapy via Integrated Cascade Reactions. Angew. Chem. Int. Ed. 60, 9480-9488 (2021).

20 Huo, M. et al. Construction of Single-Iron-Atom Nanocatalysts for Highly Efficient Catalytic Antibiotics. Small 15, 1901834 (2019).

21 Kim, M. S. et al. Heme Cofactor-Resembling Fe-N Single Site Embedded Graphene as Nanozymes to Selectively Detect $\mathrm{H}_{2} \mathrm{O}_{2}$ with High Sensitivity. Adv. Funct. Mater. 30, 1905410 (2019).

22 Jiao, L. et al. Boron-Doped Fe-N-C Single-Atom Nanozymes Specifically Boost Peroxidase-Like Activity. Nano Today 35, 100971 (2020).

23 Jiao, L. et al. Unsymmetrically Coordinated Single Fe- $\mathrm{N}_{3} \mathrm{~S}_{1}$ Sites Mimic the Function of Peroxidase. Nano Today 40, 101261 (2021).

24 Zhou, G. et al. Spin-Sate Reconfiguration Induced by Alternating Magnetic Field for Efficient Oxygen Evolution Reaction. Nat. Commun. 12, 4827 (2021).

25 Foppa, L., Coperet, C. \& Comas-Vives, A. Increased Back-Bonding Explains Step-Edge Reactivity and Particle Size Effect for CO Activation on Ru Nanoparticles. J. Am. Chem. Soc. 138, 16655-16668 (2016).

26 Gao, R. et al. Pt/Fe $\mathrm{F}_{2} \mathrm{O}_{3}$ with Pt-Fe Pair Sites as a Catalyst for Oxygen Reduction with Ultralow Pt Loading. Nat. Energy 6, 614-623 (2021).

$27 \mathrm{Hu}, \mathrm{M}$. et al. MOF-Confined Sub-2 nm Atomically Ordered Intermetallic PdZn Nanoparticles as HighPerformance Catalysts for Selective Hydrogenation of Acetylene. Adv. Mater. 30,1801878 (2018). 
28 Jiao, L. et al. From Metal-Organic Frameworks to Single-Atom Fe Implanted N-doped Porous Carbons: Efficient Oxygen Reduction in Both Alkaline and Acidic Media. Angew. Chem. Int. Ed. 57, 85258529 (2018).

29 Wei, X. et al. Highly-Defective Fe-N-C Catalysts towards pH-Universal Oxygen Reduction Reaction. Appl. Catal. B: Environ. 263, 118347 (2020).

30 Wei, X. et al. Synergistically Enhanced Single-Atomic Site Fe by Fe 3 C@C for Boosted Oxygen Reduction in Neutral Electrolyte. Nano Energy 84, 105840 (2021).

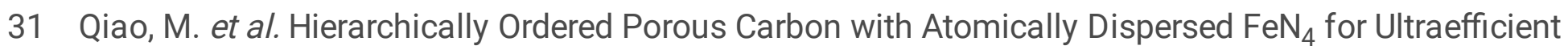
Oxygen Reduction Reaction in Proton-Exchange Membrane Fuel Cells. Angew. Chem. Int. Ed. 59, 26882694 (2020).

32 Zhang, N. et al. High-Purity Pyrrole-Type $\mathrm{FeN}_{4}$ Sites as a Superior Oxygen Reduction Electrocatalyst. Energy Environ. Sci. 13, 111-118 (2020).

$33 \mathrm{Li}, \mathrm{K}$. \& Xue, D., Estimation of Electronegativity Values of Elements in Different Valence States. J. Phys. Chem. A 110, 11332-11337 (2006).

34 Gan, G. et al. Active Sites in Single-Atom Fe-N ${ }_{x}-C$ Nanosheets for Selective Electrochemical Dechlorination of 1,2-Dichloroethane to Ethylene. ACS Nano 14, 9929-9937 (2020).

35 Han, Y. et al. Electronic Structure Engineering to Boost Oxygen Reduction Activity by Controlling the Coordination of the Central Metal. Energy Environ. Sci. 11, 2348-2352 (2018).

36 Li, Z. et al. The Marriage of the $\mathrm{FeN}_{4}$ Moiety and MXene Boosts Oxygen Reduction Catalysis: Fe 3d Electron Delocalization Matters. Adv. Mater. 30, 1803220 (2018).

$37 \mathrm{Li}, \mathrm{X}$. et al. Identification of the Electronic and Structural Dynamics of Catalytic Centers in Single-FeAtom Material. Chem 6, 3440-3454 (2020).

$38 \mathrm{Xu}, \mathrm{H}$., Cheng, D., Cao, D. \& Zeng, X. C. A Universal Principle for a Rational Design of Single-Atom Electrocatalysts. Nat. Catal. 1, 339-348 (2018).

39 Chen, Z. et al. Unraveling the Origin of Sulfur-Doped Fe-N-C Single-Atom Catalyst for Enhanced Oxygen Reduction Activity: Effect of Iron Spin-State Tuning. Angew. Chem. Int. Ed. 60, 25404-25410 (2021).

40 Cheng, W. et al. Boosting Defective Carbon by Anchoring Well-Defined Atomically Dispersed Metal- $\mathrm{N}_{4}$ Sites for ORR, OER, and Zn-air Batteries. Appl. Catal. B: Environ. 260, 118198 (2020).

41 Yang, G. et al. Regulating Fe-Spin State by Atomically Dispersed Mn-N in Fe-N-C Catalysts with High Oxygen Reduction Activity. Nat. Commun. 12, 1734 (2021). 
42 Wu, Y. et al. Defect-Engineered Nanozyme-Linked Receptors. Small 17, 2101907 (2021).

43 Xia, X. et al. Pd-Ir Core-Shell Nanocubes: a Type of Highly Efficient and Versatile Peroxidase Mimic. ACS Nano 9, 9994-10004 (2015).

44 Xi, Z. et al. Strain Effect in Palladium Nanostructures as Nanozymes. Nano Lett. 20, 272-277 (2020).

45 Travascio, P., Witting, Pa., Grant Mauk, A. \& Sen, D. The Peroxidase Activity of a Hemin-DNA Oligonucleotide Complex: Free Radical Damage to Specific Guanine Bases of the DNA. J. Am. Chem. Soc. 123, 1337-1348 (2001).

46 Ivancich, A., Dorlet, P., Goodin, D. \& Un, S. Multifrequency High-Field EPR Study of the Tryptophanyl and Tyrosyl Radical Intermediates in Wild-Type and the W191G Mutant of Cytochrome c Peroxidase, J. Am. Chem. Soc. 123, 5050-5058 (2001).

47 Christoforidis, K. C., Louloudi, M., Milaeva, E. R., Sanakis, Y. \& Deligiannakis, Y. EPR Study of a Novel [Fe-Porphyrin] Catalyst. Mol. Phys. 105, 2185-2194 (2007).

48 Chen, X. et al. Bound Oxygen-Atom Transfer Endows Peroxidase-Mimic M-N-C with High Substrate Selectivity. Chem. Sci. 12, 8865-8871 (2021).

49 Yang, Y. Q., Yang, Y. C., Liu, M. H. \& Chan, Y. H. FRET-Created Traffic Light Immunoassay Based on Polymer Dots for PSA Detection. Anal. Chem. 92, 1493-1501. (2019).

50 Diaz-Fernandez, A., Miranda-Castro, R., de-Los-Santos-Alvarez, N., Lobo-Castanon, M. J. \& Estrela, P. Impedimetric Aptamer-Based Glycan PSA Score for Discrimination of Prostate Cancer from Other Prostate Diseases. Biosens. Bioelectron. 175, 112872 (2021).

51 Lyu, Z. et al. Single-Atom Nanozymes Linked Immunosorbent Assay for Sensitive Detection of Abeta 1-40: A Biomarker of Alzheimer's Disease. Research 2020, 4724505 (2020).

52 He, J. H., Cheng, Y. Y., Zhang, Q. Q., Liu, H. \& Huang, C. Z. Carbon Dots-Based Fluorescence Resonance Energy Transfer for the Prostate Specific Antigen (PSA) with High Sensitivity. Talanta 219, 121276 (2020).

53 Jang, H. D., Kim, S. K., Chang, H. \& Choi, J. W. 3D Label-Free Prostate Specific Antigen (PSA) Immunosensor Based on Graphene-Gold Composites. Biosens. Bioelectron. 63, 546-551 (2015).

54 Kavosi, B., Salimi, A., Hallaj, R. \& Moradi, F. Ultrasensitive Electrochemical Immunosensor for PSA Biomarker Detection in Prostate Cancer Cells Using Gold Nanoparticles/PAMAM Dendrimer Loaded with Enzyme Linked Aptamer as Integrated Triple Signal Amplification Strategy. Biosens. Bioelectron. 74, 915923 (2015). 
55 Zhang, H. et al. Single Atomic Iron Catalysts for Oxygen Reduction in Acidic Media: Particle Size Control and Thermal Activation. J. Am. Chem. Soc. 139, 14143-14149 (2017).

\section{Figures}

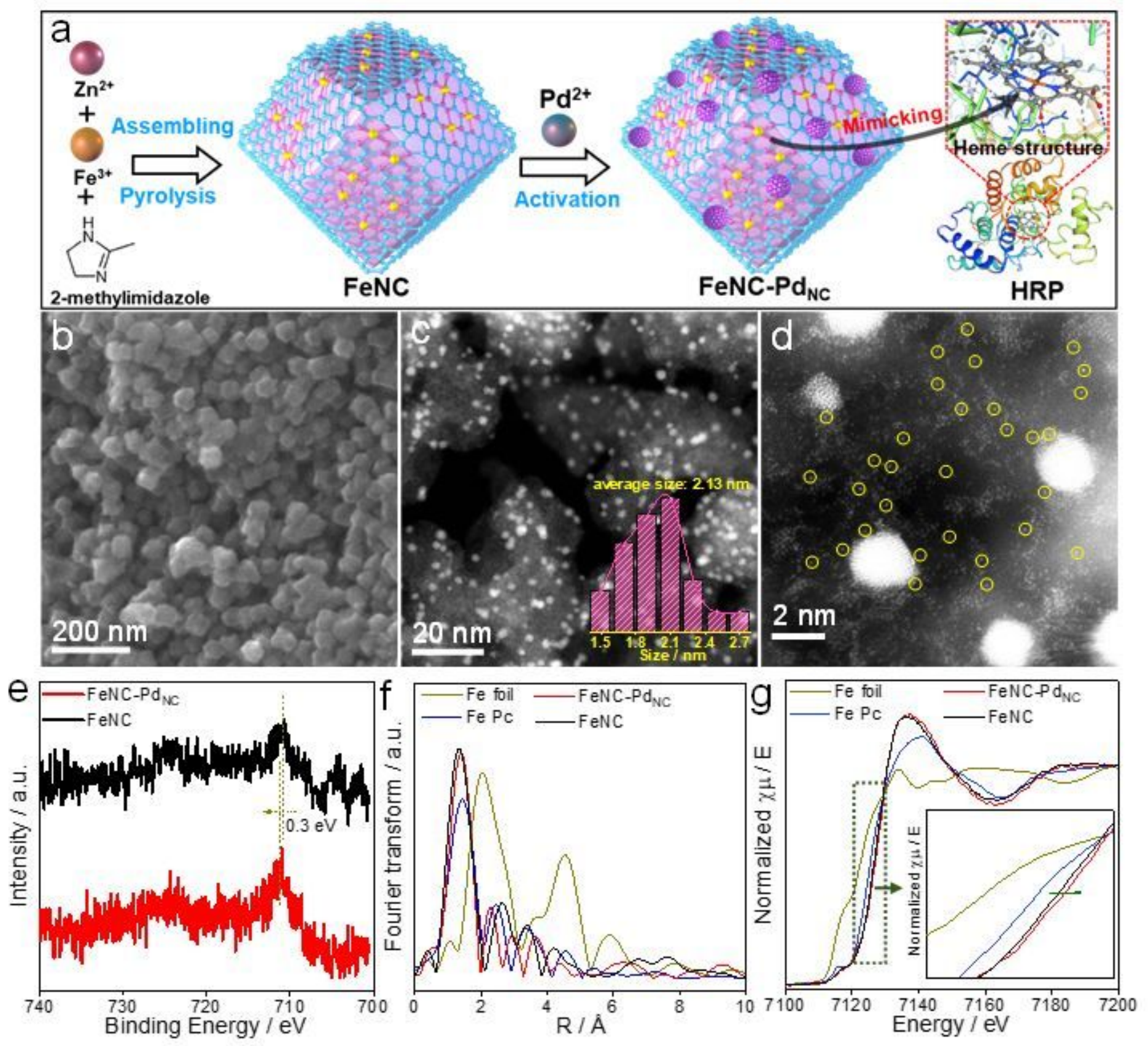

Figure 1

Synthesis and characterizations of nanozymes. (a) Schematic illustration of the synthesis of FeNC-Pd $\mathrm{NC}_{\text {. }}$

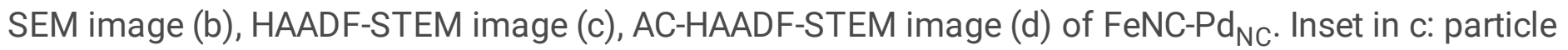
size distribution of $\mathrm{Pd}_{\mathrm{NC}}$. (e) High-resolution Fe $2 p$ XPS spectra of FeNC-Pd $\mathrm{NC}_{\mathrm{NC}}$ and FeNC. Fourier- 
transformed magnitudes of the experimental Fe K-edge EXAFS signals ( $f$ ) and XANES spectra $(\mathrm{g})$ of FeNC-Pd ${ }_{N C}, F e N C, F e$ foil, and Fe Pc. Inset in g: the enlarged spectra.

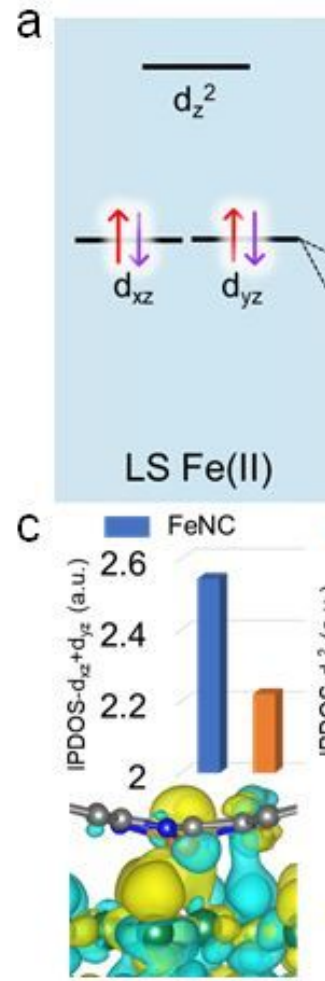

FeNC-Pd $\mathrm{NC}_{\mathrm{NC}}$
FeNC
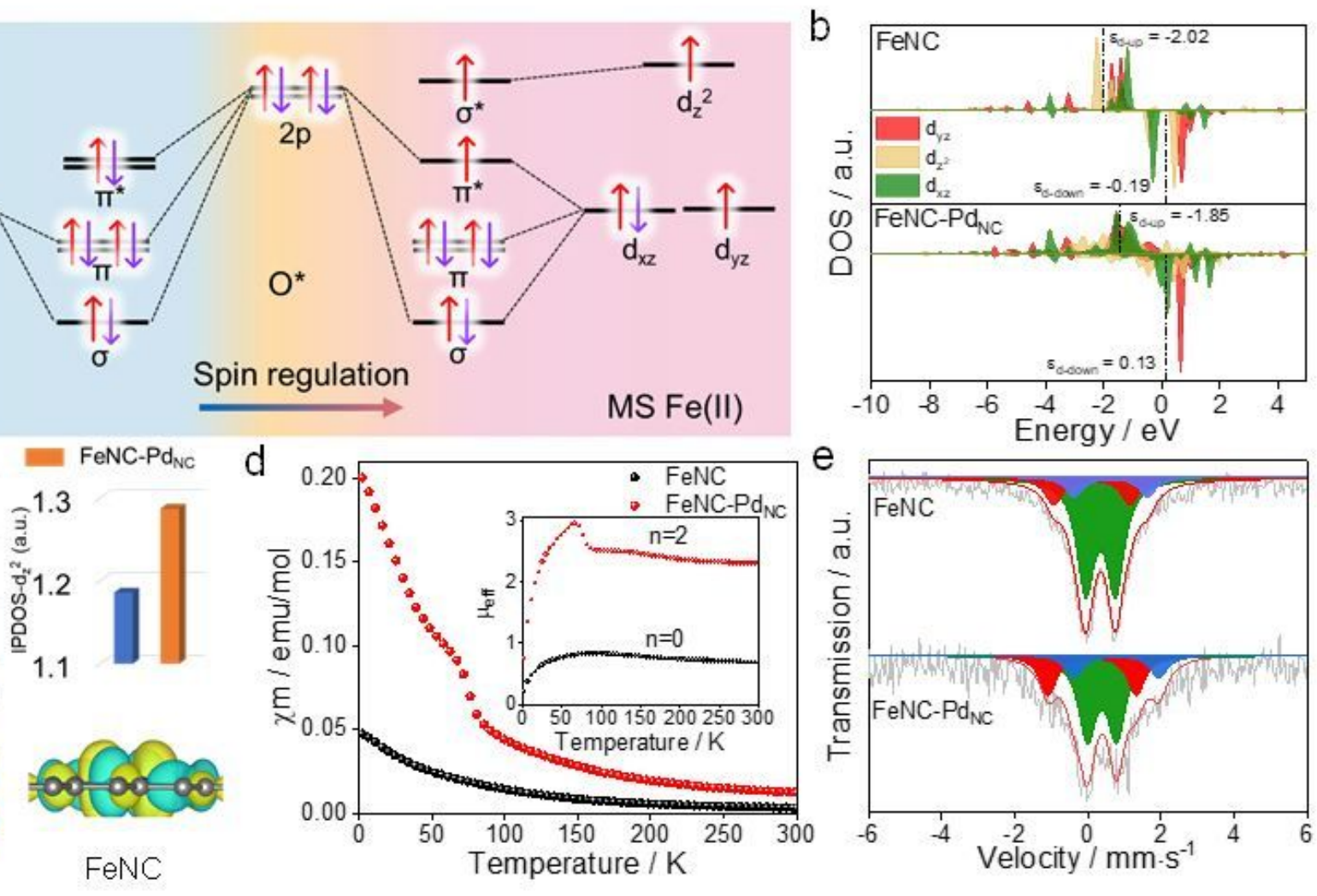

MS Fe(II)

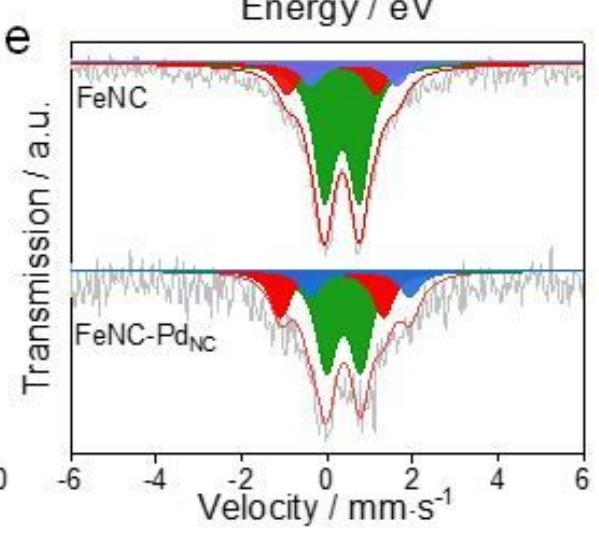

\section{Figure 2}

Spin regulation of Fe single atoms. (a) The orbital interaction between Fe single atom and ${ }^{*} \mathrm{O}$ under different spin configurations. (b) Projected DOS diagrams of FeNC and FeNC-Pd $\mathrm{NC}_{\text {. }}$ (c) The integrated crystal orbital Hamilton population (IICOHPI) values of different orbits for FeNC and FeNC-Pd $\mathrm{NC}_{\mathrm{NC}}$ (up), and the HOMO for Fe(II) in FeNC-Pd ${ }_{\mathrm{NC}}$ and FeNC (down). $\chi_{\mathrm{m}}$ plots and the $\mu_{\text {eff }}(\mathrm{d}),{ }^{57} \mathrm{Fe}$ Mössbauer transmission spectra and their deconvolution (e) of FeNC and FeNC-Pd $\mathrm{NC}_{\mathrm{NC}}$. 

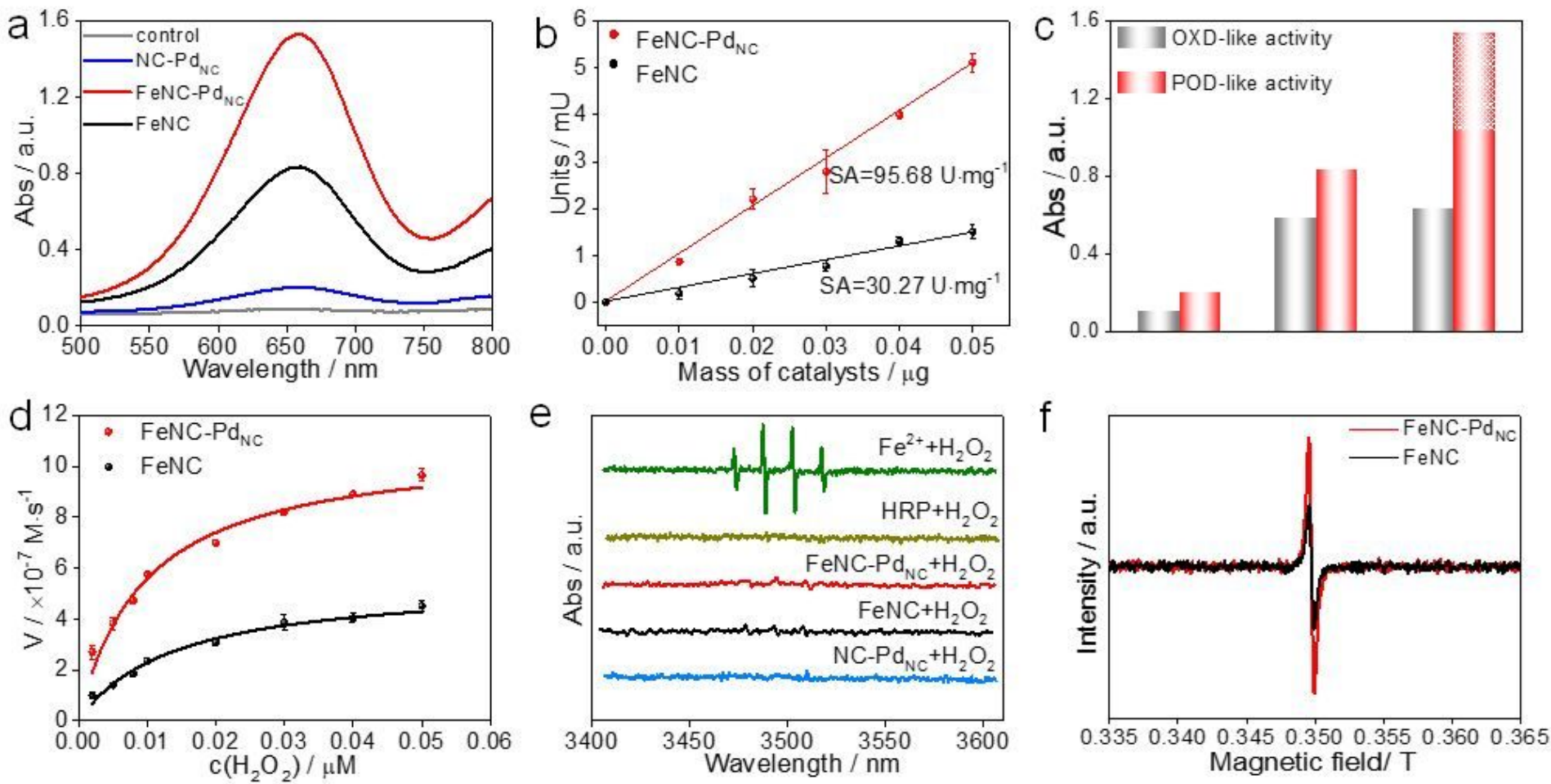

Figure 3

The POD-like performances of nanozymes. (a) UV-vis spectra of FeNC-Pd $\mathrm{NC}_{\mathrm{NC}}$, FeNC and NC-Pd $\mathrm{d}_{\mathrm{NC}}$ in the $\mathrm{TMB} / \mathrm{H}_{2} \mathrm{O}_{2}$ system. (b) Comparison of the SA of FeNC-Pd $\mathrm{NC}_{\mathrm{N}}$ and FeNC. (c) The absorbance value of oxTMB solutions at $652 \mathrm{~nm}$ in OXD-like or POD-like reaction systems using FeNC-Pd $\mathrm{NC}_{\mathrm{NC}}, \mathrm{FeNC}$ and NC-Pd $\mathrm{NC}_{\mathrm{NC}}$.

(d) Michaelis-Menten kinetic analysis of FeNC-Pd $\mathrm{NC}_{\mathrm{NC}}$ and FeNC toward different concentrations of $\mathrm{H}_{2} \mathrm{O}_{2}$.

(e) EPR spectra of $\cdot \mathrm{OH}$ produced by different systems. (f) EPR spectra of FeNC and FeNC-Pd $\mathrm{NC}_{\mathrm{N}}$ in the presence of excess $\mathrm{H}_{2} \mathrm{O}_{2}$ at $77 \mathrm{~K}$. 

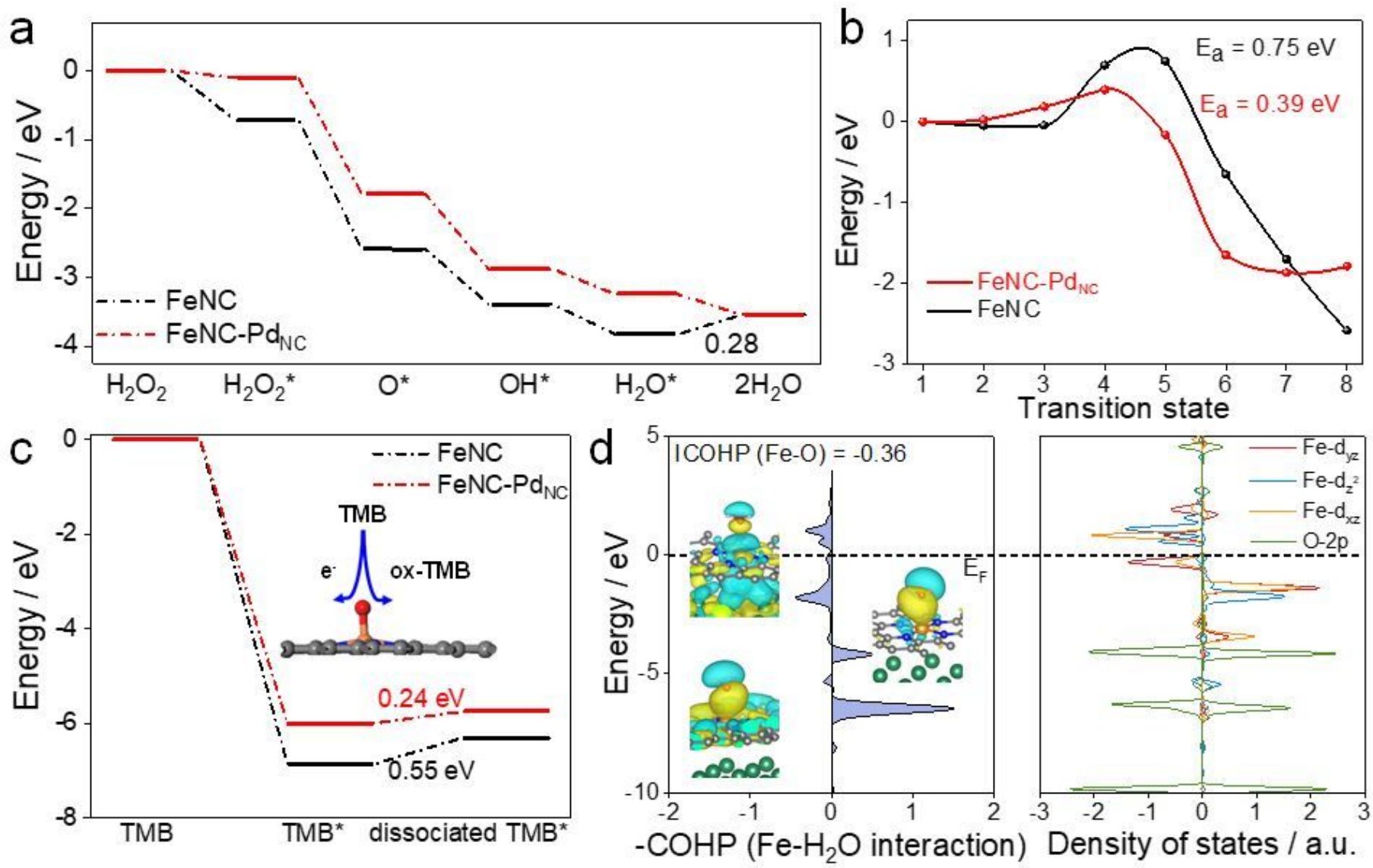

\section{Figure 4}

Proposed POD-like mechanism. (a) The free-energy diagrams for the POD-like mechanism for FeNC and FeNC-Pd $\mathrm{NC}_{\mathrm{NC}}$ (b) Calculated $\mathrm{H}_{2} \mathrm{O}_{2}$ dehydration process on FeNC-Pd $\mathrm{NC}_{\mathrm{NC}}$ and FeNC. (c) The free-energy diagrams of TMB oxidation. (d) Calculated PCOHP and DOS diagram with the $\mathrm{H}_{2} \mathrm{O}$ * on FeNC-Pd $\mathrm{NC}_{\mathrm{NC}}$. 


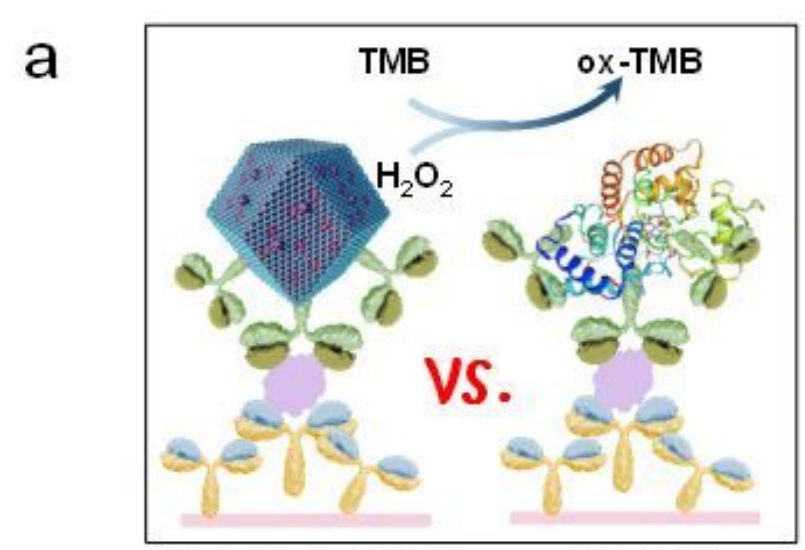

FeNC-Pd $d_{N C}$ ELISA

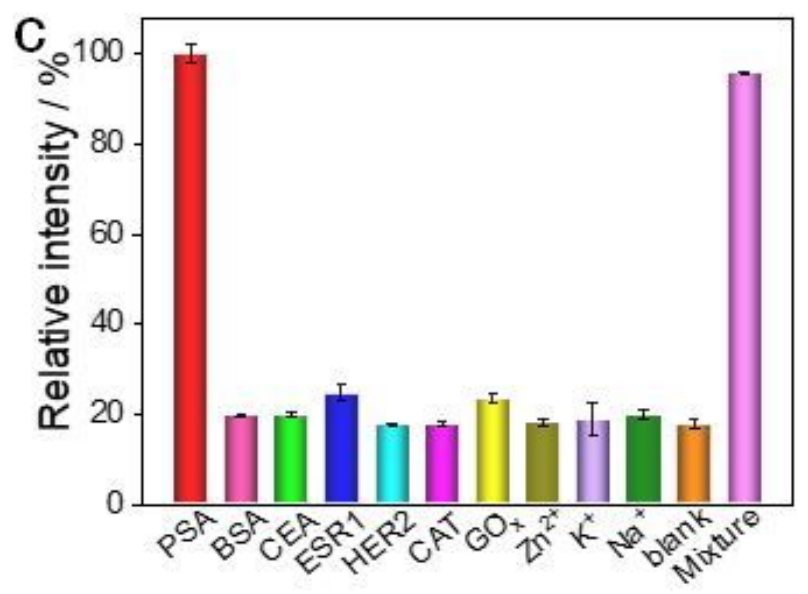

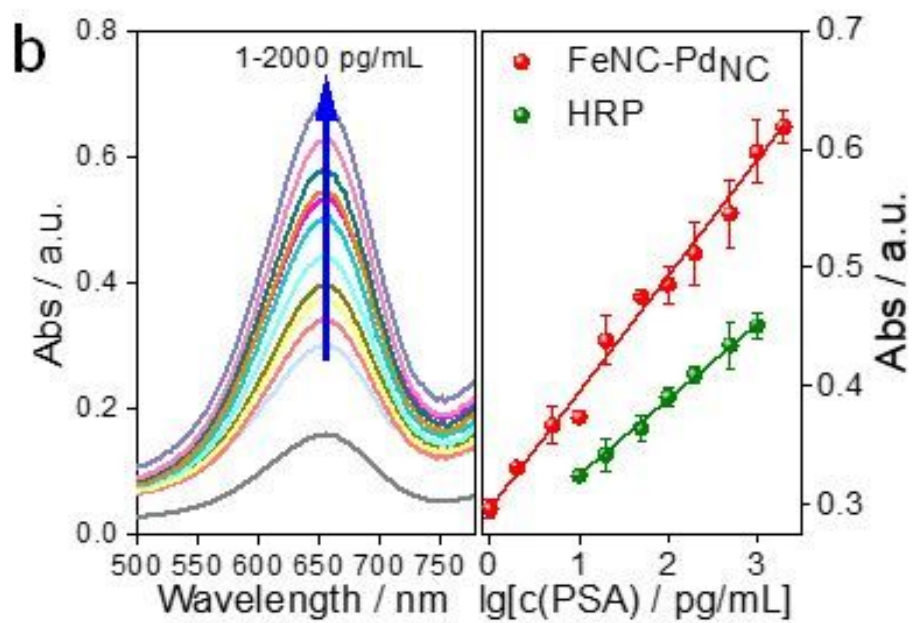

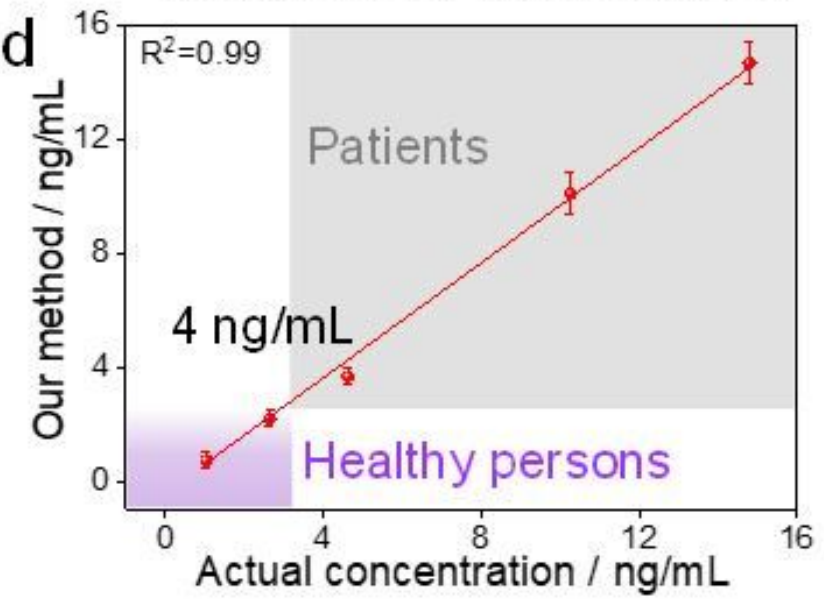

Figure 5

Application of POD mimics. (a) Schematic illustration of colorimetric detection of PSA. (b) Absorption spectra of ox-TMB with different PSA concentrations (left) and standard curves of PSA detection by using FeNC-Pd $\mathrm{NC}_{\mathrm{NC}}$ and HRP as labels (right). (c) Absorbance values for detection of different targets. (d) A linear relationship between the results of our method and the chemiluminescence for the detection of human serum samples.

\section{Supplementary Files}

This is a list of supplementary files associated with this preprint. Click to download.

- supportinginformation.docx 